INPLASY

PROTOCOL

To cite: Goren et al. Trust in government and compliance with health instructions during respiratory epidemics and pandemics: A systematic review protocol. Inplasy protocol 2021100041. doi: 10.37766/inplasy2021.10.0041

Received: 13 October 2021

Published: 13 October 2021

Corresponding author:

Talia Goren

taliagoren@gmail.com

Author Affiliation:

School of political sciences, University of Haifa.

Support: None.

Review Stage at time of this submission: The review has not yet started.

Conflicts of interest:

None declared.

\section{Trust in government and compliance with health instructions during respiratory epidemics and pandemics: A systematic review protocol}

Goren, T1; Beeri, I2; Vashdi, DR³.

Review question / Objective: We will use the ConditionContext-Population (CoCoPop) framework (Munn et al., 2015) in this review, which aims to explore the following question: Do the features of respiratory epidemics and pandemics (e.g., magnitude, duration) impact the associations between (types of) trust in government and compliance with health guidelines?

Condition being studied: The association between trust in government and authorities and civic compliance with health guidelines during respiratory epidemics and pandemics.

Eligibility criteria: 1 . Studies that explore the relationship between trust in government and authorities and civic compliance or compliance intentions with real or simulated health guidelines, during respiratory epidemics and pandemics; 2. Studies that focus on the following pandemics: avian influenza (H5N1), swine influenza (H1N1), Middle East respiratory syndrome (MERS), severe acute respiratory syndrome (SARS) and COVID-19; 3. Studies on the general healthy population (excluding health care professionals) 4. Studies that were published since 2002 to present.

INPLASY registration number: This protocol was registered with the International Platform of Registered Systematic Review and Meta-Analysis Protocols (INPLASY) on 13 October 2021 and was last updated on 13 October 2021 (registration number INPLASY2021100041).

\section{INTRODUCTION}

Review question / Objective: We will use the Condition-Context-Population (CoCoPop) framework (Munn et al., 2015) in this review, which aims to explore the following question: Do the features of respiratory epidemics and pandemics (e.g., magnitude, duration) impact the associations between (types of) trust in government and compliance with health guidelines? 
Rationale: Trust in government and in health authorities is considered a key factor in enhancing public compliance with health guidelines, particularly during health crises. However, recent studies performed during the Coronavirus pandemic, show great variance in the mere existence of the association between trust and compliance. Recent and previous studies also vary in the definition and operationalization of trust in government as well as in the tools for examining acceptance of different crisis-mitigating health behaviors. This review aims to map the conditions in which trust in government and health authorities is associated with civic compliance with health guidelines during respiratory epidemics and pandemics. It will include a specification and categorization of types of trust in government and authorities, health behaviors and features of the pandemics (e.g., magnitude, duration).

Condition being studied: The association between trust in government and authorities and civic compliance with health guidelines during respiratory epidemics and pandemics.

\section{METHODS}

Participant or population: General population; Citizens; general public.

Intervention: NA.

Comparator: NA.

Study designs to be included: Observational; Experimental; Qualitative interviews; focus groups; Quantitative surveys, cross-sectional.

Eligibility criteria: 1. Studies that explore the relationship between trust in government and authorities and civic compliance or compliance intentions with real or simulated health guidelines, during respiratory epidemics and pandemics; 2. Studies that focus on the following pandemics: avian influenza (H5N1), swine influenza (H1N1), Middle East respiratory syndrome (MERS), severe acute respiratory syndrome (SARS) and COVID-19; 3. Studies on the general healthy population (excluding health care professionals) 4 . Studies that were published since 2002 to present.

Information sources: Electronic databases; Preprints - Gray literature; Consultation with leading researchers in the field; Major journals; Citation tracking - Cited by/ Reference search.

Main outcome(s): Identifying the conditions under which trust in government and health authorities may be related to civic compliance with health guidelines during a health crisis, may assist policy makes adjust and promote policies and behaviors that are suitable for these conditions and consequently maximize their effect on civic compliance. This is not only a practical contribution, but also a theoretical contribution to policy design literature.

Quality assessment / Risk of bias analysis: We plan to use the Mixed Methods Appraisal Tool (MMAT) Version 2018. The MMAT is designed for the appraisal stage of systematic mixed studies reviews. It allows to appraise the methodological quality of the following study types: qualitative research, randomized controlled trials, non-randomized studies, quantitative descriptive studies, and mixed methods studies.

Strategy of data synthesis: We will apply a narrative synthesis approach and begin with the "vote-count" technique in order to identify and report the number of studies in which the association between trust in government and health authorities and civic compliance with health guidelines was significant. Next, we will describe and group the analyzed studies by the main examined features of the study (e.g., study design, type pf trust, pandemic type and magnitude, geographic location of the studies' execution and type of instruction(s)). The synthesized insights will be presented textually, tabularly and in other formats of graphic visualization.

Subgroup analysis: NA. 
Sensitivity analysis: NA.

Language: No restriction.

Country(ies) involved: Israel.

Keywords: Trust in government; Trust in authorities; Compliance; Health guidelines;

Pandemics; Health crises.

Contributions of each author:

Author 1 - Talia Goren.

Author 2 - Itai Beeri.

Author 3 - Dana Rachel vashdi. 